\title{
Review of: "Assessing central serous chorioretinopathy with deep learning and multiple optical coherence tomography images"
}

\author{
Qiang Chen $^{1}$ \\ 1 Nanjing University of Science and Technology
}

Potential competing interests: The author(s) declared that no potential competing interests exist.

This paper gave a model for CSC classification with OCT images by combining deep learning technique and traditional machine learning technique. The technique novelty and the clinical meaning is limited. The main problems are as follows:

1. The "Assessing" in the title is not very proper, because this paper only did the "classification".

2. The related work on the classification of retinal diseases was not introduced in this paper.

3. The model performance listed in Table 2 can't be used to represent the best classification performance, because the advanced deep learning technologies were not adopted in this paper.

4. The inputs of Fig. 3 and Fig. 4 are not consistent with the real training phase because the authors only used lesion cuts, not the all 25 SD-OCT images.

5. In Data preprocessing, how to "cropped the $596 \times 1264$-pixel SD-OCT images and obtained $380 \times 764$ pixel red-green-blue (RGB) images"?

6. In this paper, the acute and chronic CSC images should be provided and analyzed, which will make readers understand the difference between them better.

7. As said by the authors "the number of subjects was not large enough and external validation was not performed", the clinical meaning of this study is limited.

8. In reference, the latest two years' study was missed. 